\title{
PERFORMANCE ANALYSIS OF ML AND MMSE DECODING USING GRADIENT BASED ALGORITHM
}

\author{
K.Mahalakshmi ${ }^{1}$, S.Palanivelrajan ${ }^{2}$ \\ ${ }^{I} P G$ Scholar, Communication systems, M.Kumarasamy College of Engineering, Karur, India \\ ${ }^{2}$ Assistant Professor, Electronics and Communication Engineering, M.Kumarasamy College of Engineering, Karur, India
}

\begin{abstract}
Discrete-time all-analog-processing joint source-channel coding with Maximum Likelihood (ML) and Minimum Mean Square Error (MMSE) detections are employed in fast fading Rayleigh channel. Co-operative communication achieves spatial diversity using single antenna devices. Spatial diversity improves the performance of analog joint source-channel coding in wireless fading channels. In the case of discrete time processing signals ML decoding is unstable when compared to MMSE. Thus gradient based algorithm is proposed to reduce the complexity of the $M L$ decoding. This algorithm quantizes the discrete processing signals into a continuous process. It also increases the capacity and cost of the channels thus making it suitable for the multiple input multiple output (MIMO) systems.
\end{abstract}

Keywords: Analog joint-source channel coding, gradient based algorithm, Cooperative communications. $* * *$

\section{INTRODUCTION}

Wireless systems brought a constructive evolution to the modern communication era. They are preferred over wired systems due to various factors like improved mobility and reliability. The information is subjected to encoding and modulation while the reverse processes of decoding and demodulation occurs at the receiver side. The two important wireless system considerations include power and bandwidth efficiency besides security. The security in wireless systems is ensured by cryptographic strategies whereas the efficient utility of bandwidth is promoted by source and channel coding strategies thus enabling optimum performance of the system. A digital system is optimal based on the source and channel separation.

Co-operative diversity involves sharing of antenna and other resources enabling a creation of virtual array [2]. A novel cooperative source channel technique is proposed in which the correlation between sources is utilized for optimum transmission [3]. Neji et al. analyzed application of L-DACS to future aeronautical systems. Further a self-repairing system with a two layer architecture that could provide appreciable scalability and reasonable fault coverage was proposed [4].Discrete-time continuous-amplitude sources based analog systems are proposed an alternative for analog systems. Considering the transmit power in a wireless system there are two factors that affect the transmit power to a large extend. One is more power requirement for transmission of both user information's. The other one is that the user will require less power due to diversity gains. Numerous research literatures are proposed to throw limelight on coding for signal manipulation in wireless communication systems. These literatures demystify the coding strategies in wireless systems.

Figure.1 consists of the block diagram of wireless communication. It consists of the source which is then encoded by the channel encoder. Then the channel is

Decoded by the channel decoder and passed to the message sink. The wireless channel is provided with the Bit Error Rate (BER) and the Rate Controller.

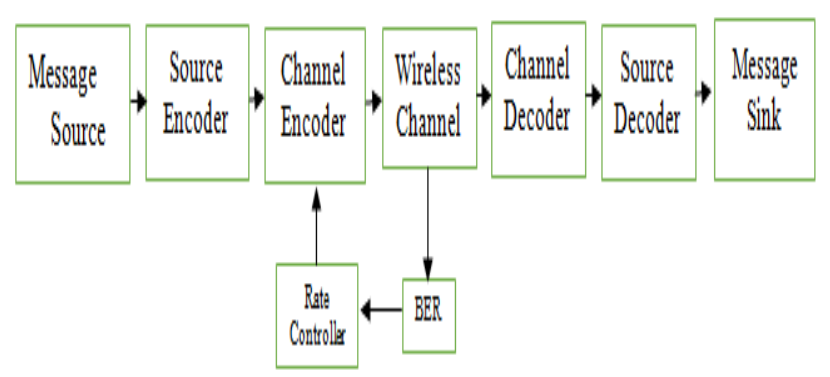

Fig -1: Block diagram of Wireless Communication

The demand has increased for high data-rate transmission, in the recent wireless mobile communications. One of the most promising techniques is the MIMO which is used to increase the data-rate and system capacity, because it can effectively take advantage of the random fading.

Maximum likelihood detection is the optimal signal detection for the MIMO system, which can achieve the minimum BER. The minimum BER is the need for the system. 
However, MLD requires a prohibitively large amount of computational complexity that exponentially increases with both the number of data streams and that of constellations. The complexity can be reduced by the suboptimal detection algorithm. The zero-forcing (ZF) algorithm requires a very small amount of complexity but, owing to the noise enhancement it exhibits poor BER performance. This degradation can be alleviated to a certain extent by the MMSE algorithm but it cannot achieve sufficient BER performance.

This paper proposes a new suboptimal algorithm for the MIMO signal detection that limits the use of the linear detection without the Eigen value decomposition. The proposed algorithm sets the initial guess to the solution of $\mathrm{ZF}$ or MMSE and then searches signal candidates in the direction of a modified gradient vector of the metric, which can reduce the search space. Also, the proposed algorithm outperforms the conventional ones of which complexity order is equal to that of ZF.

This article deals with related works in Section II. Section III focuses on decoding techniques and section IV briefs the results and analysis is performed with the depicted results. The paper is concluded in section $\mathrm{V}$.

\section{RELATED WORKS}

A lot of research articles are directed to concentrate on coding techniques for wireless communication systems. Hu et al. [1] analyzed Source-Channel Coding Using Non-Linear Curves and MMSE Decoding. Apart from coding, transmission efficiency can also be enhanced by use of Diversity techniques since in a wireless channel different users undergo varying time dependent attenuation.

Kim et al. [15] proposed a gradient based algorithm which utilises sub optimal maximum likelihood detection for MIMO channels. The projected algorithm considers signals that are transmitted as continuous variables similar to the conventional method destined for the discrete optimization problem, and examines components of the transmitted signals in the direction of gradient vector of the specified metric. The proposed work assumes MIMO system model with $\mathrm{N}_{\mathrm{T}}$ antennas for transmission and $\mathrm{N}_{\mathrm{R}}$ receive antennas. The channel is presumed to be a time- invariant flat fading channel. The BER performance of the algorithm for 16 QAM and QPSK are analyzed and verified with results.

Zhu et al. [7] analyzed the performance of the maximum likelihood detection in MIMO systems. A tight union bound for the probability of symbol error rate (SER) for the proposed MIMO-MLD systems with asymptotic representation is proposed and two dimensional signal constellations (QAM and PSK) is proposed. With the proposed bound, metrics such as channel estimation, constellation size, and antenna configuration are evaluated. In this literature, all the symbols are assumed to be equiprobable. The performance comparison of 16 QAM and PSK are performed with emphasis on simulation results for BLAST and MLD.

Damen et al. [13 ] proposed maximum likelihood detection for MIMO systems with focus on selection of the closest lattice points. In involves evolution of two novel algorithms. The first of the two algorithms is encouraged by the Pohst enumeration strategy and it also renders a notorious improvement in complexity reduction compared to the Viterbo-Boutros sphere decoder. The association between the proposed algorithm and stack sequential decoding algorithm is proven. The computational complexity of various algorithms are compared with supportive contributions from pre-processing and ordering and it is also inferred that the proposed decoder achieves performance appreciable to that of MLD and the connection between sphere decoding and sequential decoding is established and simulation results are analyzed.

\section{SYSTEM MODEL}

Consider the transmission of data in analog form from a discrete-time memory less Gaussian source. The system has a continuous-amplitude and transmission is done over a wireless channel shown in figure.2. The transmitter side symbolizes the presence of, $\mathrm{N}$ i.i.d. source symbols which are encoded into $\mathrm{K}$ channel symbols respectively. Further they are transmitted through a fast-fading Rayleigh channel. The assumption is that transmitted source symbols have zero mean with and average power of symbols is $\sigma_{\mathrm{h}}{ }^{2}$.

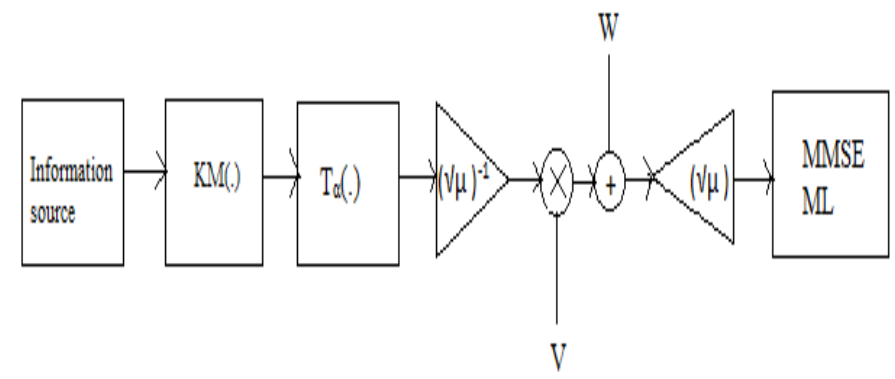

Fig -2: System model for wireless communications

The mean square error [MSE] is accordingly is given by equation [1].

$$
\text { MSE }=\frac{1}{N}\|X-\dot{X}\|
$$


MSE is used to evaluate distortion of $\dot{X}$ with respect to $\mathrm{X}$.

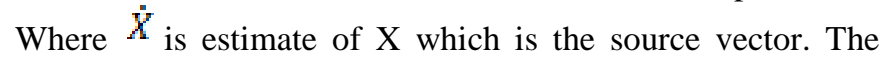
measurement of system performance is given by plotting signal to distortion ratio [SDR] against channel signal to noise ratio [CSNR]. The encoder has a function KM (.) that could map the source vector into $\mathrm{K}$ dimensional surface. The normalization factor is given by $\mathrm{T}_{\alpha}$. The surface lies in the $\mathrm{N}$ dimensional surface which is scaled by $\Delta$. To attain enhanced flexibility in the discussed scheme an invertible function is manipulated, followed by application of a normalization factor.

\section{DECODING TECHNIQUES}

Source samples $\mathrm{X}=\left(\mathrm{x}_{1}, \mathrm{x}_{2}\right)$ are encoded using the Archimedes' spiral in a 2:1 bandwidth reduction system. The pair of coordinates is represented as shown below in equation (1) and (2).

$$
\begin{gathered}
x=\operatorname{sign}(\theta) \frac{\Delta}{\pi} \theta \sin \theta \\
x_{\theta, 2}=\frac{\Delta}{\pi} \theta \cos \theta
\end{gathered}
$$

Where $\Delta$ symbolizes the two distances between neighboring Arms of the spiral, and $\theta$ is the angle between origin and point say $\mathrm{X}$.

\subsection{Decoder}

It is simplest decoding method. In ML decoding, the inverse function $T^{-1}($.) is performed primarily and again it is inverse mapped.

\subsection{MMSE Decoder}

Considering the code-book $\mathrm{C}$ assuming it to be composed of a set of $\mathrm{m}$ code-words, i.e. $=\mathrm{x}^{1} ; \mathrm{x}^{2} ; \ldots ; \mathrm{x}^{\mathrm{m}}$, then the maximum likelihood de-coder chooses, as estimated input, the most probably code-words ${ }^{\mathrm{k}}$ given are received output and it is attained by maximizing $\mathrm{P}\left(\mathrm{x}^{\mathrm{k}}=\mathrm{y}\right)$. This method is complex and cannot be applied to $3: 1$ and 4:1 systems.

\subsection{Gradient Based Algorithm}

The general problem of minimizing a function is, with respect to a set of parameters is at the root of many issues in wireless communication. Gradient based learning draws on the fact that it is generally much easier to minimize a reasonably smooth, continuous function than a discrete function. The loss function can be minimized by estimating the impact of small variations of the parameter values on the loss function. This is measured by the gradient of the loss function with respect to the parameters.
Efficient learning algorithms can be devised when the gradient vector can be computed analytically. This is the basis of numerous gradient based learning with continuous valued parameters. The set of parameters $\mathrm{W}$ is a real valued vector.

In the simplest case, e is a scalar constant. More sophisticated procedures use variable e, or substitute it for a diagonal matrix, or substitute it for an estimate of the inverse matrix. The conjugate gradient method is used.

$$
W_{k}=W_{k-1}-e \frac{\partial E(W)}{\partial W}
$$

A popular minimization procedure is the gradient algorithm, also called the on-line update. It consists in updating the parameter vector using a noisy, or approximated version of the gradient vector. In the most common instance of it $\mathrm{W}$ is updated, on the basis of the single sample:

$$
W_{k}=W_{k-1}-e \frac{\partial E^{\rho k}(W)}{\partial W}
$$

With this procedure the parameter vector fluctuates around an average trajectory, but usually converges considerably faster than regular gradient descent and second order methods on large training sets with redundant samples (such as those encountered in speech or character recognition).

\section{SIMULATION RESULTS}

The performance of ML and MMSE for Rayleigh fading scenarios under single antenna system is depicted in figure. 3 . The result shown below symbolizes that both ML and MMSE performs similar in Rayleigh fading under low signal to distortion ratio (SDR) values. The parameters considered for comparison are CSNR and SDR. The results imply that ML is preferred since it presents similar SDR performance results but with the advantage of reduced complexity. MMSE decoder utilizes channel statistics in decoding performance and performance ML is inferior to MMSE if CSI is present only at the receiver side.

Similarly performance of the ML and MMSE are analyzed for $2: 1$ system in Rayliegh fading channels is depicted in figure. 3 which shows almost similar performance attribute to one antenna system 


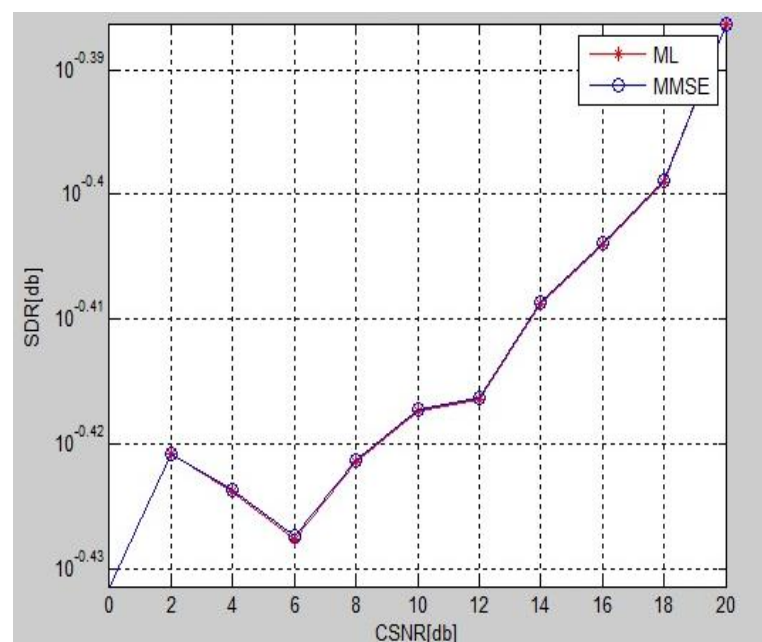

Fig -3: Performance of ML \& MMSE in Rayliegh fading

The difference in performance is well pronounced in cooperative transmission as shown in figure.4. The performance of MMSE is predominant in cooperative communication scenario as ML decoder aims at improving the estimation error thus providing optimal performance but lesser performance compared to MMSE. In a cooperative scenario, mean square error minimization technique improves system performance compared to likelihood technique as it involves decreasing the estimation error for a given value of CSNR.

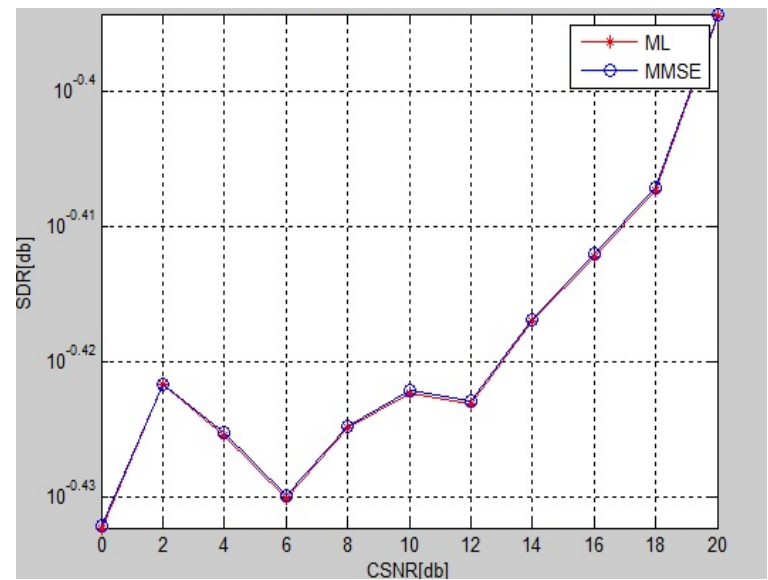

Fig -4: Performance of ML \& MMSE in Rayliegh fading condition for 2:1 system

The performance of protocols under various bandwidth reduction systems are depicted and analyzed in figure.5. Interestigly the protocols perform optimally in 2:1, 3:1 systems when compared to $1: 1$ systems. The performance is similar in cases of 2:1, 3:1 systems implying that these protocols are best suited for these sytems compared to other systems

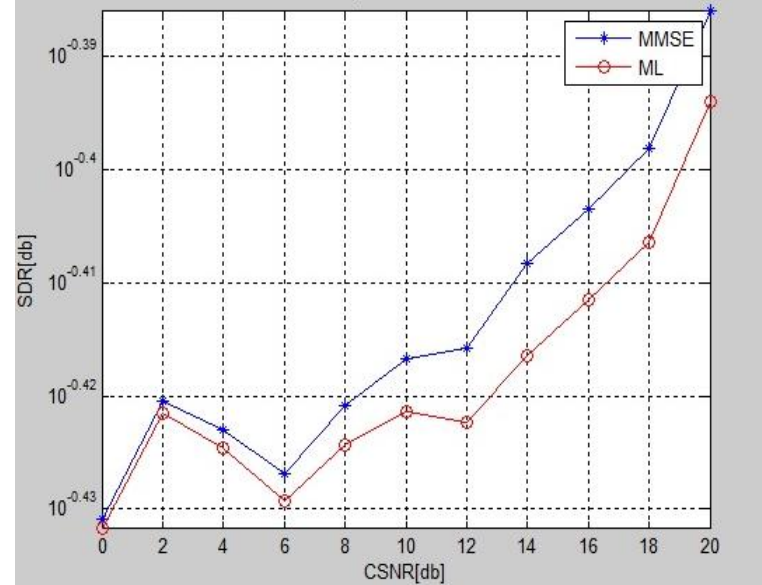

Fig -5: Performance comparison in cooperative scenario

The performance of protocols under various bandwidth reduction systems are depicted and analyzed in figure.4.

Interestigly the protocols perform optimally in 2:1, $3: 1$ systems when compared to $1: 1$ systems. The performance is similar in cases of 2:1, 3:1 systems implying that these protocols are best suited for these sytems compared to other systems.

In figure. 6 we analyse the performance of $\mathrm{ml}$ and mmse decoding using the gradient based algorithm. The result shown below symbolizes that both ML and MMSE performs similar in Rayleigh fading under low signal to distortion ratio (SDR) values. The parameters considered for comparison are CSNR and SDR. The results imply that ML is preferred since it presents similar SDR performance results but with the advantage of reduced complexity. MMSE decoder utilizes channel statistics in decoding performance and performance ML is inferior to MMSE if CSI is present only at the receiver side.

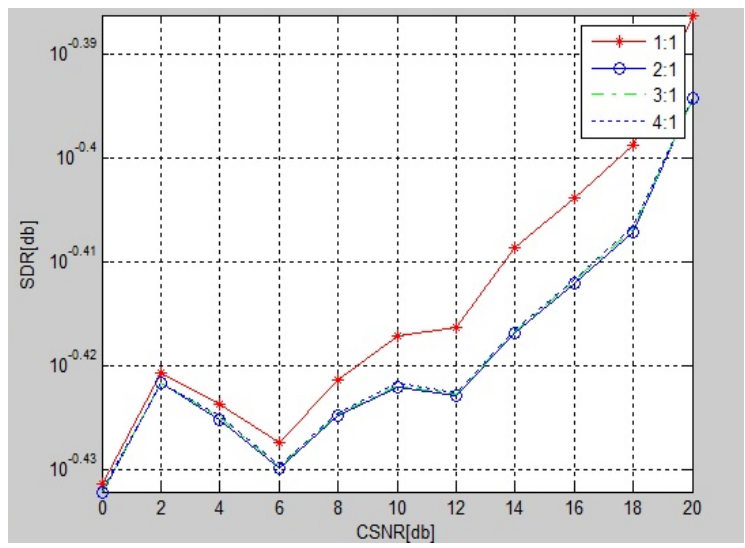

Fig -6: Performance of protocols for various bandwidth reduction systems 
Thus there is an enhancement in the result. The complexity of $\mathrm{ml}$ decoding is reduced which also reduces the cost. The performance of $\mathrm{ml}$ and mmse is almost similar in the single antenna system.

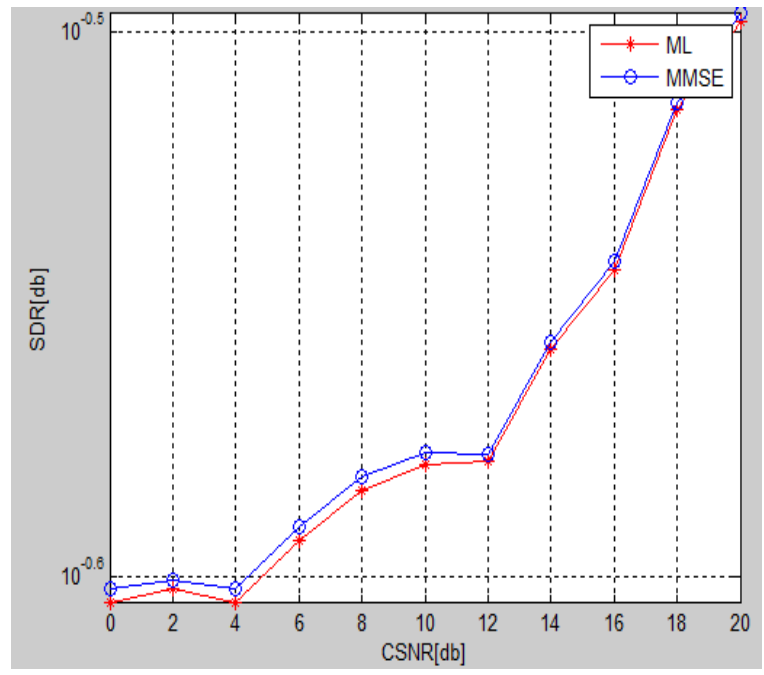

Fig -7: Performance comparison using Gradient based algorithm

The performance is much more effective in using the gradient based algorithm since it chooses the corrrect receivers and performs the results. This is a main advantage of the algorithm in order to perform in a better way. Thus the results are performed for MIMO systems.

The existing system consits of the 2:1 and 3:1 systems for which the results are analysed.In this system for multiple inputs multiple outputs are considered using the algorithm. Thus the performance results in a much more effective way when compared to the performance of the existing systems. The main aim of the proposed systen is to reduce the complexity and it is achieved to the desired rate which also reduces the cost.

In figure.7 the performance comparison is made for ML and MMSe decoding in co operative communication which is done by using gradient based algorithm. The complexity of ML decoding is reduced and it is also made to be stable.

Figure.8 indicates the performance comparison in 2:1 systems. The complexity of ML is reduced to the maximum in this system.

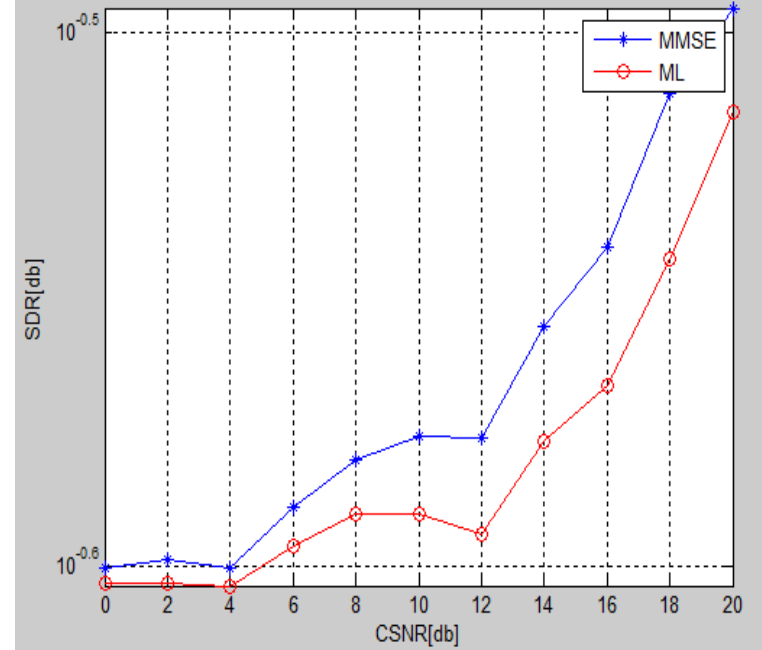

Fig -8: Performance comparisons in the cooperative systems using gradient based algorithm

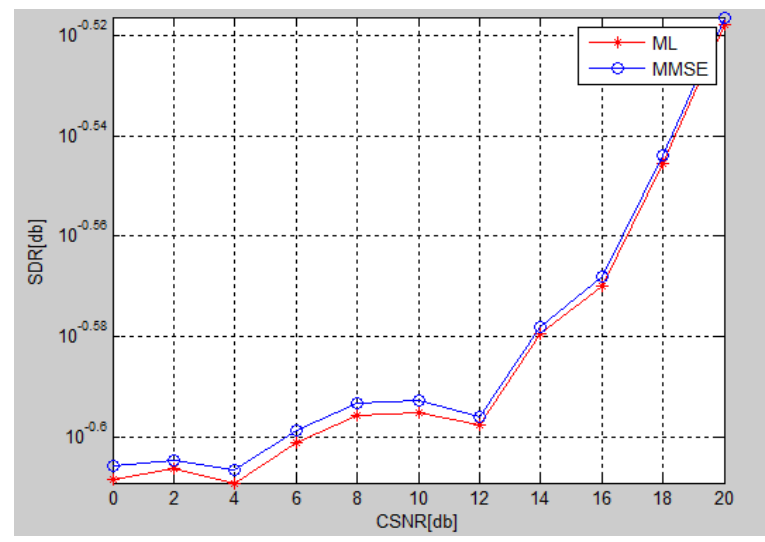

Fig -9: Performance comparision in the 2:1 systems

\section{CONCLUSIONS}

A discrete-time all-analog processing joint source-channel coding scheme is considered for analysis and results for under various scenarios are presented for discussion. A Rayleigh fading channel is considered for performance comparison of MMSE and ML and it is inferred that for single antenna system ML and MMSE perform equal and also in a 2:1 system they perform almost equal. Finally MMSE performs better than $\mathrm{ML}$ in the co operative communication.

The proposed system consists of gradient based algorithm which quantizes the discrete signals into continuous signals and reduces the complexity and makes the system stable which reduces the cost of the system.

In future work some other algorithm can be used to increase the performance of ML decoding so that in will used in a better way. 


\section{REFERENCES}

[1]. Glauber Brante, Richerd Demo Souza and Javier GraciaFrias, "Spatial diversity Using analog joint source channel Coding In wireless Channels," IEEE Transactions On CommunicationS, Vol. 61, No. 1, January 2013.

[2]. NajettNeji,Raul de Lacerda,E, Alain Azoulay,ThierryLetertre\& Olivier Outtier Survey on, "The Future Aeronautical Communication System and Its Development for Continental Communications," IEEE Transactions On Vehicular Technology, Vol. 62, No. 1, January 2013.pp 182-191.

[3]. Yichuan Hu, Javier Garcia-Frias, MeritxellLamarca, "Analog Joint Source-Channel Coding Using Non Linear Curves and MMSE Decoding," IEEE Transactions On Communications, Vol. 59, No. 11, November 2011 pp 30163025.

[4]. Sung Hoon Lim, Young-Han Kim, Abbas El Gamal,SaeYoung Chung, "Noisy Network Coding," IEEE Transactions On Information Theory, Vol. 57, No. 5, May 2011.pp 3132- 3151

[5]. Fredrik Hekland,Pal Anders Floor,and Tor A. Ramstad, "Shannon-Kotel'nikov Mappings in Joint Source-Channel Coding," IEEE Transactions On Communications, Vol. 57, No. 1, January 2009.pp 94-105

[6]. DenizGündüz, and ElzaErkip, "Source and Channel Coding for Cooperative Relaying," IEEE Transactions On Information Theory, Vol. 53, No. 10, October 2007.pp 34543475

[7]. X. Li and X. Cao, "Low complexity signal detection algorithm for MIMO-OFDM systems," IEE Electronics Letters, vol. 41, no. 2, Jan. 2005

[8]. J. Nicholas Laneman, David N. C. Tse Gregory W. Wornell, "Cooperative Diversity in Wireless Networks: Efficient Protocols and Outage Behavior," IEEE Transactions On Information Theory, Vol. 50, No. 12,pp 3062-3079 December 2004.

[9]. A. Nosratinia, T. E. Hunter, and A. Hedayat, "Cooperative communication in wireless networks," IEEE Commun. Mag., vol. 42, no. 10, pp. 74-80, Oct. 2004.

[10]. K. Honjo and T. Ohtsuki, "Computational complexity reduction of MLD based on SINR in MIMO spatial multiplexing systems," IEEE Vech Tech. Conf. vol. 2, pp. 1426-1430, Sept. 2004.

[11]. Arul D. Murugan, Praveen K. Gopala\&Hesham El Gamal, "Correlated Sources Over Wireless Channels: Cooperative Source-Channel Coding IEEE Journal On Selected Areas In Communications," Vol. 22, No. 6, August 2004.pp 988-998.

[12]. K. Fukawa, Y. Yamada, H. Suzuki, and S. Suyama, "A MIMO-OFDM maximum likelihood reception scheme in cochannel interference environments," IEEE Vehi. Tech. Conf., vol. 4, pp. 1849-1853, May 2004.

[13]. M. O. Damen, H. E. Gamal, and G. Caire, "On maximum-likelihood detection and the search for the closest lattice point," IEEE Trans. Info. Theory, vol. 49, no. 10, pp. 2389-2402, Oct. 2003

[14]. V. Pammer, Y. Delignon, W. Sawaya, and D. Boulinguez, "A low complexity suboptimal MIMO receiver: the combined ZF-MLD algorithm," IEEE PIMRC 2003, pp. 2271-2275, Sept. 2003

[15]. K. J. Kim and J. Yue, "Joint channel estimation and data detection algorithms for MIMO-OFDM systems," ThirtySixth Asilomar Conference on Signals, Systems and Computers, pp. 1857-1861, Nov. 2002

\section{BIOGRAPHIES}

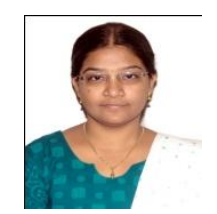

PG Scholar in M.Kumarasamy Engineering College 